\title{
Aortic balloon valvuloplasty as a bridge-to-decision in patients with aortic stenosis
}

\author{
Jacek Wacławski ${ }^{1}, K_{\text {Krzysztof Wilczek }}{ }^{1}$, Bartosz Hudzik ${ }^{1,2}$, Damian Pres ${ }^{1}$, Michał Hawranek ${ }^{1}$, \\ Krzysztof Milewski ${ }^{3}$, Piotr Chodór ${ }^{4}$, Michał Zembala ${ }^{5}$, Mariusz Gąsior ${ }^{1}$
}

${ }^{1} 3^{\text {rd }}$ Department of Cardiology, School of Medicine with the Division of Dentistry in Zabrze, Medical University of Silesia in Katowice, Silesian Centre for Heart Disease in Zabrze, Poland

${ }^{2}$ Department of Cardiovadcular Disease Prevention, School of Public Health in Bytom, Medical University of Silesia in Katowice, Poland ${ }^{3}$ Center for Cardiovascular Research and Development, American Heart of Poland, Katowice, Poland

${ }^{4} 1^{\text {st }}$ Department of Cardiology, School of Medicine with the Division of Dentistry in Zabrze, Medical University of Silesia in Katowice, Silesian Centre for Heart Disease in Zabrze, Poland

${ }^{5}$ Department of Cardiac, Vascular and Endovascular Surgery and Transplantology in Zabrze, Medical University of Silesia in Katowice, Poland

Adv Interv Cardiol 2019; 15, 2 (56): 195-202

DOI: https://doi.org/10.5114/aic.2019.86012

\section{A bstract}

Introduction: Balloon aortic valvuloplasty (BAV) is a method of treatment for patients who are temporally ineligible for surgical aortic valve replacement (AVR) or transcatheter aortic valve implantation (TAVI). This procedure allows one to select patients with severe left ventricle dysfunction or with symptoms of unknown origin who can benefit from AVR or TAVI.

Aim: To evaluate the efficacy, safety and outcome of therapy in patients treated with balloon aortic valvuloplasty. To define clinical characteristics, immediate and distant outcomes of the procedure, and factors affecting the 12-month mortality.

Material and methods: We retrospectively evaluated the procedural and clinical outcomes of 47 consecutive patients with severe, symptomatic aortic stenosis (AS) who underwent balloon aortic valvuloplasty in our center.

Results: Age and logistic EuroSCORE were $76.81 \pm 6.64$ and $22.85 \pm 13.74$, respectively. The mean gradient after the procedure decreased from $52.23 \pm 18.21$ to $35.52 \pm 13.43 \mathrm{~mm} \mathrm{Hg}(p=0.001)$. Major complications occurred in $5(10.6 \%)$ patients. In-hospital, 30-day and 1-year mortalities were $6.38 \%, 10.63 \%$ and $42.55 \%$, respectively. $31.9 \%$ of patients underwent the destination therapy (TAVI or AVR). One-year mortality in the group treated conservatively after BAV was $56.2 \%$, while in the group treated with AVR or TAVI it was $13.3 \%$. Procedural success, presence of arterial hypertension, and performance of the destination therapy were factors associated with a decreased 1-year mortality.

Conclusions: Balloon aortic valvuloplasty should be treated as a bridge-to-decision on further treatment. Balloon aortic valvuloplasty has high efficacy and an acceptable adverse events rate. Patients undergoing balloon valvuloplasty are high-risk patients with many comorbidities.

Key words: aortic stenosis, transcatheter aortic valve implantation, aortic valve replacement, aortic balloon valvuloplasty.

Su m mary

Balloon aortic valvuloplasty (BAV) is a method of treatment for patients who are temporally ineligible for surgical aortic valve replacement or transcatheter aortic valve implantation. Our aim was to evaluate the efficacy, safety and outcome of therapy in patients treated with balloon aortic valvuloplasty. We retrospectively evaluated the procedural and clinical outcomes of 47 consecutive patients with severe, symptomatic aortic stenosis who underwent balloon aortic valvuloplasty in our center. In conclusion, BAV should be treated as a bridge-to-decision on further treatment. Balloon aortic valvuloplasty has high efficacy and an acceptable adverse events rate. Patients undergoing balloon valvuloplasty are high-risk patients with many comorbidities.

\section{Corresponding author:}

Jacek Wacławski MD, PhD, $3^{\text {rd }}$ Department of Cardiology, School of Medicine with the Division of Dentistry, Medical University of Silesia, Silesian Centre for Heart Disease, 9 M. Skłodowskiej-Curie St, 41-800 Zabrze, Poland, phone: +48 505132140, e-mail: jacek.waclawski@gmail.com Received: 29.03.2018, accepted: 22.01.2019. 


\section{Introduction}

Balloon aortic valvuloplasty (BAV) was first implemented in 1985 as a treatment method for patients with severe aortic stenosis, who were disqualified from a surgical procedure due to concomitant diseases. The BAV leads to the extension of aortic cusps and annulus, microfractures of valve calcifications, and a partial separation of the commissures. After the procedure a vast majority of patients report clinical improvement. Nevertheless, it has been confirmed that BAV does not improve the survival rate; usually the stenosis recurs after several months [1-3]. Following transcatheter aortic valve implantation (TAVI) introduction, and as the population ages, a significant rise in the frequency of aortic valvuloplasty is observed, which is not only an integral part of the TAVI procedure, but also a bridging strategy. Balloon aortic valvuloplasty can be also considered as a palliative therapy in individual cases, in which due to severe comorbidities there are contraindications to surgery, and it is impossible to perform the TAVI procedure [4].

\section{Aim}

The aim of the study was to evaluate the efficacy, safety and outcome of therapy in patients treated with balloon aortic valvuloplasty and to define clinical characteristics, immediate and distant outcomes of the procedure and factors affecting the 12-month mortality.

\section{Material and methods}

We retrospectively evaluated the procedural and clinical outcomes of 47 consecutive patients with severe, symptomatic aortic stenosis (AS) who underwent balloon aortic valvuloplasty in our center. Severe AS was diagnosed on the basis of echocardiographic examination with aortic valve area calculated from the continuity equation less than $1 \mathrm{~cm}^{2}\left(<0.6 \mathrm{~cm}^{2}\right.$ per $1 \mathrm{~m}^{2}$ of the body surface area). Each patient was analyzed individually, considering their complete clinical picture. All patients were symptomatic with considerably impaired exercise tolerance, reporting stenocardia, with prior syncope or documented ventricular tachycardia. Qualification for the BAV procedure was performed during a Heart Team meeting.

Patients requiring improvement of hemodynamic conditions who at the moment of the decision did not qualify for the surgical treatment or TAVI were qualified for this procedure. The procedure was performed due to two indications: (1) as a bridge therapy before aortic valve replacement (AVR) or TAVI, or as a bridge-to-decision concerning further treatment, (2) as a palliative therapy. BAV was planned as a stand-alone procedure, while coronary angio and percutaneous coronary intervention ( $\mathrm{PCl}$ ) (if needed) were performed prior to valvular intervention.
Balloon aortic valvuloplasty was performed in the cath lab with OR backup by an experienced operator. The transvenous temporary pacing electrode was introduced into the right ventricle in all patients. The decision concerning the application of the Swan-Ganz catheter was left to the discretion of the operator. Puncture of femoral artery was performed using the Seldinger technique and a vascular sheath (9-11 F) was introduced (one diameter larger sheath makes the balloon removal easier and safer). Subsequently, the Amplatz L1 catheter was passed through the aortic valve. The pressure gradient through the aortic valve was measured. Next, after the replacement of the guide wire and the arterial sheath a valvuloplasty balloon (BALTON) was introduced (the size had been selected on the basis of annulus dimensions evaluated in echocardiographic examination; length of the balloon was $40 \mathrm{~mm}$ ) in the area of the aortic valve. During a rapid stimulation $(180 / \mathrm{min})$ repeated inflation of the balloon was performed. According to the operator's experience a few shorter inflations are more effective in terms of increasing the AVA without additional side effects or the risk of hemodynamic compromise due to prolonged rapid pacing. The result of the procedure was evaluated on the basis of hemodynamic parameters and echocardiographic examination. A procedure in which the aortic valve area calculated from the continuity equation or the Gorlin formula increased by $40 \%$ or the mean gradient on the aortic valve decreased by $40 \%$ was recognized as an effective one.

The primary endpoints of the study were death and qualification for destination therapy (TAVI or AVR). Secondary endpoints were major complications, defined as peri-procedural death, myocardial infarction, stroke, acute severe aortic regurgitation, need for pacemaker implantation, major vascular complications, life-threatening or major bleeding, acute kidney injury stage 2 and 3, conversion to AVR, classified according to the Valve Academic Research Consortium-2 (VARC-2) criteria [5].

Depending on the patient's clinical condition, the results of the obtained invasive and non-invasive examinations, and comorbidities, the patient was eventually qualified for conservative treatment, TAVI, or the surgical AVR.

\section{Statistical analysis}

Quantitative data are given as means and standard deviations. Qualitative data are displayed as frequencies. The Shapiro-Wilk test was employed to determine whether random samples came from a normal distribution. The $\chi^{2}$ test with Yates' correction was used to compare categorical variables. The unpaired $t$-test was used to compare normally distributed continuous variables. One-year survival was estimated with the KaplanMeier method and compared with the log-rank test. The effects of the clinical variables on the 1-year mortality were assessed using the multivariate Cox proportional 
hazard regression models with the results expressed as hazard ratios (HRs) and 95\% confidence intervals ( $\mathrm{Cls}$ ). Variables with a significant influence on mortality in univariate analysis were entered into the multivariate model. A $p$-value of less than 0.05 was considered statistically significant. Statistical analyses were performed using the Statistica 7 software package.

\section{Results}

The procedure of the percutaneous balloon aortic valvuloplasty was performed in 47 subjects with severe symptomatic aortic stenosis. In the bridge group there were 24 subjects, and in the group of palliative care there were 23 patients.

$A$ vast majority of patients were elderly, with numerous concomitant diseases. A considerable proportion of the group that qualified for the procedure were patients after myocardial infarction, with previous coronary artery bypass graft (CABG) and with neurological disorders.

All patients qualified for the BAV procedure were symptomatic, with $85 \%$ of patients having NYHA functional class of III or IV; while syncope appeared in 15\% of patients. Baseline clinical characteristics of the study group are presented in Tables I and II.

After completion of the balloon valvuloplasty procedure, the maximum gradient on the aortic valve decreased from $92.2 \pm 31.2$ to $61.4 \pm 22.8 \mathrm{~mm} \mathrm{Hg}(p<0.001)$, the mean gradient from $52.2 \pm 18.2$ to $35.5 \pm 13.4 \mathrm{~mm} \mathrm{Hg}$ $(p=0.001)$. The aortic valve area (AVA) increased from $0.54 \pm 0.18$ to $0.8 \pm 0.22 \mathrm{~cm}^{2}(p<0.001)$. The increase of AVA by $>40 \%$ was achieved in $36(76 \%)$ patients. The left ventricle ejection fraction after the procedure did not change significantly compared to the initial value. Patients with previous severe mitral regurgitations showed more significant clinical improvement after the procedure (mostly reduction of shortness of breath).

Similarly to the echocardiographic analysis, a significant improvement of hemodynamic parameters was observed. Prior to the procedure, the value of the maximum gradient equaled $107.8 \mathrm{~mm} \mathrm{Hg}$, and the mean gradient was $54 \mathrm{~mm} \mathrm{Hg}$. After the procedure a significant reduction of the maximum gradient to $70.8 \mathrm{~mm} \mathrm{Hg}$ ( $p=$ $0.025)$ was observed, and the mean gradient decreased to $34 \mathrm{~mm} \mathrm{Hg}(p<0.001)$. The aortic valve area increased from 0.61 to $0.93 \mathrm{~cm}^{2}(p<0.0021)$. The procedure of the percutaneous balloon aortic valvuloplasty was effective according to the assumed criteria in 40 (85.1\%) patients.

The BAV procedure was performed with the application of a single balloon with the mean diameter of 21 $\pm 2.29 \mathrm{~mm}$, and with its repeated inflation, performed $3.69 \pm 1.28$ times on average. The procedure lasted 112 $\pm 24 \mathrm{~min}$ on average. The radiation dose reached 0.84 \pm 0.62 Gy and the quantity of the contrast medium was $86.66 \pm 78.29 \mathrm{ml}$. In two subjects the procedure was performed with no contrast medium administered (kidney
Table I. Baseline characteristics of patients

\begin{tabular}{|c|c|}
\hline Parameter & $\begin{array}{c}\text { Mean } \pm \text { SD or } \\
n(\%)\end{array}$ \\
\hline Age & $76.8 \pm 6.6$ \\
\hline Female & $23(48.9)$ \\
\hline BMI & $27.5 \pm 6.2$ \\
\hline BSA & $1.8 \pm 0.19$ \\
\hline EuroSCORE & $10.6 \pm 2.3$ \\
\hline Logistic EuroSCORE & $22.8 \pm 13.7$ \\
\hline Diabetes mellitus & $22(46)$ \\
\hline Hypertension & $26(55.3)$ \\
\hline Dyslipidemia & $13(27.6)$ \\
\hline Cancer & $8(17.0)$ \\
\hline COPD & $6(12.7)$ \\
\hline PAD & $8(17.0)$ \\
\hline History of TIA & $1(2.1)$ \\
\hline History of stroke & $5(8.5)$ \\
\hline Dementia & $3(6.3)$ \\
\hline Chronic kidney disease & $18(38.2)$ \\
\hline Dialysis & $1(2.1)$ \\
\hline Coronary artery disease & $30(63.8)$ \\
\hline History of myocardial infarction & $13(27.6)$ \\
\hline History of cardiac surgery & $10(21.2)$ \\
\hline History of PCl & $14(29.7)$ \\
\hline Porcelain aorta & $2(4.2)$ \\
\hline Implanted pacemaker & $7(14.8)$ \\
\hline Sinus rhythm & $34(72.3)$ \\
\hline Atrial fibrillation & $10(21.2)$ \\
\hline NYHA I & 0 \\
\hline NYHA II & $6(12.7)$ \\
\hline NYHA III & $26(55.3)$ \\
\hline NYHA IV & $14(29.7)$ \\
\hline $\operatorname{CCS} 1$ & $3(6.3)$ \\
\hline CCS II & $8(16.9)$ \\
\hline CCS III & $6(12.7)$ \\
\hline CCS IV & $1(2.1)$ \\
\hline History of syncope & $8(17.0)$ \\
\hline
\end{tabular}

$B M I$ - body mass index, BSA - body surface area, CCS - Canadian Cardiovascular Society, COPD - chronic obstructive pulmonary disease, NYHA - New York Heart Association, $P A D$ - peripheral artery disease, $P C I$ - percutaneous coronary intervention, TIA - transient ischemic attack. 
dysfunction, no real need of contrast during BAV, used previously for diagnostic procedures). A vascular sheath with the mean diameter of $9.75 \pm 1.03 \mathrm{Fr}$ was applied. The sheath was removed with the application of mechanical pressure applied for $15 \mathrm{~min}$.

No death occurred during the periprocedural period. During the first month $3(6.4 \%)$ patients died; two of them had been admitted to hospital in cardiogenic shock. The first patient died on the $24^{\text {th }}$ day after BAV as a result of hemorrhagic complications during surgical aortic valve replacement. The second patient underwent AVR on the $13^{\text {th }}$ day after the balloon valvuloplasty, and died on the $23^{\text {rd }}$ day due to a progressive circulatory failure. The third patient died on the $8^{\text {th }}$ day after BAV as a result of a progressive circulatory failure.

Major complications occurred in 5 (10.6\%) patients. Major vascular complications (according to VARC-2) occurred in $4(8.5 \%)$ patients, and minor ones in 4 (8.5\%) patients. Most often it was a pseudoaneurysm at the

Table II. Echocardiographic parameters prior to BAV

\begin{tabular}{|c|c|}
\hline Parameter & $\begin{array}{c}\text { Mean } \pm \text { SD or } \\
n(\%)\end{array}$ \\
\hline LVEF & $35.9 \% \pm 14.7$ \\
\hline $\mathrm{RV}[\mathrm{mm}]$ & $30.1 \pm 4.6$ \\
\hline IVSDd [mm] & $13.3 \pm 2.3$ \\
\hline IVSSd [mm] & $17.6 \pm 3.3$ \\
\hline LVPWDd [mm] & $12.5 \pm 2.4$ \\
\hline LVPWSd [mm] & $18.7 \pm 10.1$ \\
\hline LVEDd [mm] & $54.6 \pm 9.0$ \\
\hline LVESd [mm] & $40.8 \pm 10.8$ \\
\hline $\mathrm{EDV}[\mathrm{ml}]$ & $133.5 \pm 61.2$ \\
\hline $\mathrm{ESV}[\mathrm{ml}]$ & $90.0 \pm 62.3$ \\
\hline Aortic root diameter [mm] & $33.9 \pm 5.2$ \\
\hline Aortic annulus diameter [mm] & $21.9 \pm 2.1$ \\
\hline $\mathrm{RVSP}[\mathrm{mmHg}]$ & $55.8 \pm 13.6$ \\
\hline $\mathrm{LA}[\mathrm{mm}]$ & $45.2 \pm 10.5$ \\
\hline Moderate MR & $16(34.8)$ \\
\hline Severe MR & $5(10.8)$ \\
\hline Moderate TR & $13(28)$ \\
\hline Severe TR & $1(2.3)$ \\
\hline
\end{tabular}

EDV - end diastolic volume, ESV - end systolic volume, IVSDd - intraventricular septum diastolic diameter, IVSSd - intraventricular septum systolic diameter, LA - left atrium, LVEDd - left ventricular end-diastolic diameter, LVEF - left ventricular ejection fraction, LVPWDd - left ventricular posterior wall end diastolic diameter, LVPWSd - left ventricular posterior wall end systolic diameter, MR - mitral regurgitation, $R V$-right ventricle, RVSP-right ventricular systolic pressure, $T R$ - tricuspid regurgitation. puncture site. A large hematoma requiring transfusion of the packed red cells occurred in 2 patients. In 1 patient a cardiac tamponade occurred, which was treated by a pericardial puncture.

A concomitant myocardial infarction, stroke, and the need of a conversion to a surgical procedure occurred in 1 patient. There were no cases of conduction disorders that would require a cardiac pacemaker insertion. In the post-procedural period deterioration of kidney function was observed in 5 patients, with an increase of creatinine level to $150-200 \%$ of the initial value. The percentage of complications is presented in Table III.

\section{Destination therapy}

Of all studied patients $31.9 \%$ were subjected to the destination therapy (surgical aortic valve replacement or transcatheter aortic valve implantation); TAVI or AVR was applied in $56.5 \%$ of patients in the bridge treatment group (the rest of the group was treated conservatively mostly due to lack of consent or concomitant diseases) and $8.35 \%$ in the palliative care group.

The destination procedure was performed 91 days after BAV on average ( 96 days for AVR, 88 days for TAVI).

In the group of patients subjected to the destination therapy a considerable reduction of 1-year mortality rate

Table III. Peri-procedural complications of BAV

\begin{tabular}{lc}
\hline Procedure complications & $\boldsymbol{N}(\%)$ \\
\hline Major complications & $5(10.6)$ \\
\hline Intra-procedural death & 0 \\
\hline In-hospital death & $3(6.4)$ \\
\hline Myocardial infarction & $1^{*}(2.1)$ \\
\hline Stroke & $1^{*}(2.1)$ \\
\hline Acute severe AR & 0 \\
\hline Vascular complications: & $8(17)$ \\
\hline Major & $4(8.5)$ \\
\hline Minor & $4(8.5)$ \\
\hline Bleeding: & $7(14.9)$ \\
\hline Life-threatening & $1(2.1)$ \\
\hline Major & $3(6.4)$ \\
\hline Minor & $3(6.4)$ \\
\hline Need for pacemaker implantation & 0 \\
\hline AKI stage 1 & 5 \\
\hline AKI stage 2 and 3 & 0 \\
\hline Conversion to AVR & $1^{*}(2.1)$ \\
\hline Ocured sinutaneously the same patient AR aotic & \\
\hline
\end{tabular}

${ }^{*}$ Occurred simultaneously in the same patient. AR - aortic regurgitation, AKIacute kidney injury. 
was observed compared to the group of patients who were subjected to the balloon valvuloplasty only $(13.3 \%$ vs. $56.2 \%$ respectively).

The data relating to mortality and the destination therapy are collected in Figures 1 and 2 .

\section{Multivariate analysis}

The multivariate analysis of the risk of 1-year death revealed that a higher mean gradient on the aortic valve prior to the procedure, female sex, presence of neurological disorders and peripheral arterial disease, were independent factors of 1-year mortality. The procedural success, presence of arterial hypertension (in our opinion it is a statistical bias), and performance of the destination therapy were factors that reduced the mortality in the examined group. The results of the multiple factor analysis are presented in Table IV.

\section{Discussion}

\section{Methodology of the procedure}

Since the introduction of the BAV procedure in 1985 , the equipment used during the procedure has evolved, and a number of improvements have been implemented in the procedure itself. The most important ones include: (1) application of lower-profile balloons allowing for the use of a smaller-diameter vascular sheath; (2) quick simulation technique during inflation of the balloon, enabling one to position it in a precise manner; (3) possibility of using vascular closure devices facilitating the maintenance of haemostasis; (4) improvement of the guide wires applied during the procedure, decreasing the risk of traumatization during the passage through the valve and inside the left ventricle; (5) new imaging techniques, including transoesophageal echocardiography (TEE) and computed tomography (CT), allowing for a more precise evaluation of the dimensions of the ring, the valve, and the anatomic relations. The majority of these contemporary improvements were utilized in the analyzed procedures.

\section{Complications}

Over the years a drop in the number of complications connected with BAV procedures has been observed. It is related to the evolution of the instruments, changes in the methodology of the procedure, and the introduction of devices intended for maintenance of vascular haemostasis. The percentage of complications over the first years after the introduction of the BAV procedure equaled $20-25 \%$. According to the latest reports over the recent years it decreased to $6.8-15.6 \%$. Similarly, the percentage of deaths relating to the procedure decreased from $3-5 \%$ to $1-2.5 \%$ and the percentage of vascular complications from $5-11 \%$ to $1.5-7 \%$ [2, 6-14].

Over the years, authors of works documenting the effects of valvuloplasty were not using uniform defini-

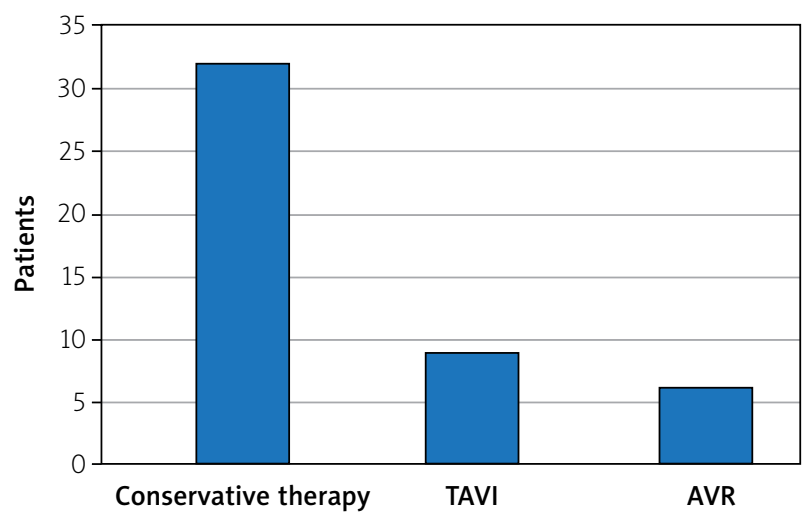

Figure 1. Destination therapy in patients after BAV

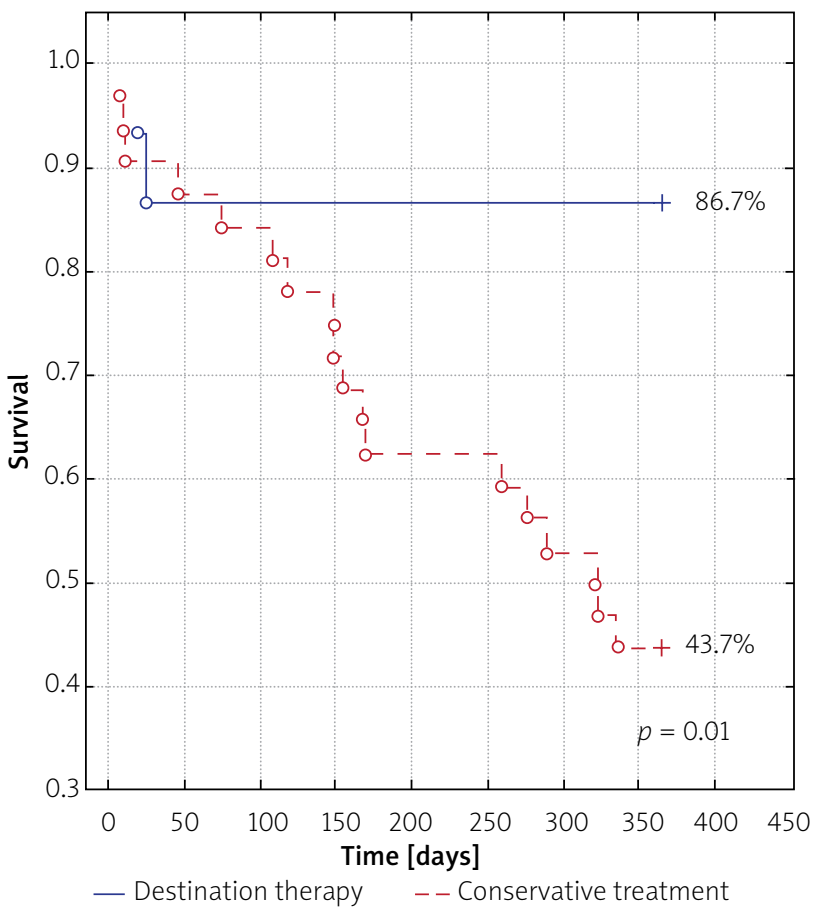

Figure 2. Kaplan-Meier estimated survival at 12 months after BAV

tions of vascular and hemorrhagic complications; hence a direct comparison of the results is impossible. Uniform definitions of vascular complications were introduced in 2011 on the basis of the works by the Valve Academic Research Consortium [15].

Major complications in our study occurred in 5 (10.6\%) patients, which corresponds to the latest reports pertaining to the results of BAV; it is a much smaller percentage than in the studies from the first period after the introduction of the balloon valvuloplasty. None of the patients died in the perioperative period.

In 1 patient from our study there was a release of embolic material (probably from the valve), which resulted in embolization of the coronary artery and a stroke of the central nervous system. An immediate conversion to AVR + CABG was performed in this patient, with a good 
Table IV. Multivariate analysis of the risk of 1-year death

\begin{tabular}{llcc} 
Parameter & HR & 95\% Cl & $P$-value \\
\hline LVEDd $[\mathrm{mm}]$ & 0.99 & $0.88-1.10$ & 0.86 \\
\hline Mean AV gradient prior to BAV $[\mathrm{mm} \mathrm{Hg}]$ & 1.09 & $1.00-1.20$ & 0.04 \\
\hline AVA index prior to BAV $\left[\mathrm{cm}^{2} / \mathrm{m}^{2}\right]$ & 5.56 & $0.80-9.33$ & 0.13 \\
\hline LVEF prior to BAV $[\%]$ & 1.07 & $0.90-1.12$ & 0.88 \\
\hline Mean AV gradient after BAV $[\mathrm{mm} \mathrm{Hg}]$ & 0.93 & $0.83-1.04$ & 0.22 \\
\hline AVA index after BAV $\left[\mathrm{cm}^{2} / \mathrm{m}^{2}\right]$ & 0.31 & $0.01-10.33$ & 0.86 \\
\hline LVEF after BAV $[\%]$ & 0.90 & $0.77-1.06$ & 0.24 \\
\hline Procedural success & 0.11 & $0.01-0.88$ & 0.037 \\
\hline Female sex & 16.3 & $1.50-25.52$ & 0.021 \\
\hline Age $[$ years] & 0.91 & $0.77-1.04$ & 0.17 \\
\hline Diabetes & 0.61 & $0.10-3.43$ & 0.57 \\
\hline Hypertension & 0.049 & $0.008-0.29$ & 0.0009 \\
\hline Dyslipidemia & 0.27 & $0.03-2.40$ & 0.24 \\
\hline PAD & 32.5 & $2.12-73.87$ & 0.012 \\
\hline Neurological disorders & 5.73 & $1.23-26.77$ & 0.026 \\
\hline Chronic kidney disease & 2.05 & $0.30-13.7$ & 0.45 \\
\hline Destination therapy (AVR/TAVI) & 0.022 & $0.001-0.29$ & 0.003 \\
\hline
\end{tabular}

effect. Major vascular complications occurred in 4 (8.5\%) subjects. It is slightly more than in the latest reports. This may result from the fact that vascular closure devices were not applied in the examined group.

The percentage of major complications, such as myocardial infarction, stroke, and conversion to a cardiac surgical procedure was low, which corresponds to the results of other studies [2, 7, 13]. Other major complications including acute aortic regurgitation, ring fracture, or necessity to implant a cardiac pacemaker, did not occur in the examined group. The percentage of their occurrence described in the subject literature was 1-2.6\%, $0.3 \%$, and $0.6-4 \%$, respectively $[2,7,9-14]$.

\section{Balloon aortic valvuloplasty as a bridge to further treatment}

In the examined group BAV constituted a useful tool enabling a bridging therapy and preparation of the patient for the destination therapy in selected cases. After the procedure of aortic valvuloplasty, TAVI was performed in $9(19.1 \%)$ subjects, AVR in 6 (12.8\%) subjects, a repeated BAV in one subject. Some patients initially ineligible for the valve replacement procedure or its transcatheter implantation, after the BAV procedure may improve their clinical condition enough to become potential candidates for the destination therapy $[10,16,17]$. Thanks to rapid clinical improvement after balloon valvuloplasty up to
$70 \%$ of patients can be qualified for surgical treatment or TAVI [18-20]. After the balloon aortic valvuloplasty a clinical assessment and qualification for a further therapy should be performed each time.

In subjects who exhibit a high operational risk and symptoms of unknown origins BAV allows one to predict clinical improvement after TAVI that could be performed. It is important, taking into account the considerable risk and costs connected with the destination procedure. Valvuloplasty can be also performed in patients ineligible for TAVI/AVR due to comorbidities, including antineoplastic treatment. In this group of subjects BAV can bring a temporary improvement while waiting for further treatment [13].

Currently, numerous researchers emphasize the role of BAV as a procedure enabling one to prepare the patient and to qualify the patient for further treatment. In the study carried out by Eltchaninoff et al. Balloon aortic valvuloplasty served as a bridge to AVR/TAVI in $26.3 \%$ of patients (AVR 9.6\%, TAVI 16.7\%) [9], and in the work by Saia et al. in $34.1 \%$ (8.6 and $25.5 \%$, respectively) [13]. These are groups comparable with the group described in our study (31.9\%). In works which describe the treatment before the introduction of TAVI, BAV served as a bridging therapy to AVR in $27-30 \%$ of cases. Considering the fact that the effects of BAV are only temporary, it is crucial that the destination therapy be performed not later than 
6-7 months after BAV. In the examined group the time to the destination therapy was shorter: it was 88 days to TAVI and 96 days to BAV.

In the study by Saia et al. $28 \%$ of patients initially disqualified from the surgical procedure were subjected to the target treatment thanks to the clinical improvement after the performance of BAV [18]. These results confirm the thesis that careful selection of patients treated by $\mathrm{BAV}$ as a bridge-to-decision may have a positive impact on the improvement in the survival of patients.

\section{Study limitations}

The present work is a retrospective analysis based on the treatment outcomes from one centre. The number of patients is lower compared to the largest published works in the world. The profile of the treated patients differs from those described in other studies, especially in the ejection fraction, which may affect the results, including mortality. The study does not have any control group.

However, it seems that these limitations do not significantly affect the main message of the study, which is to demonstrate the aim of using percutaneous balloon valvuloplasty in selected patients with severe aortic valve stenosis.

\section{Conclusions}

Patients subjected to balloon aortic valvuloplasty are high-risk patients, with numerous aggravating diseases. The procedure of balloon aortic valvuloplasty is characterized by high efficacy and an acceptable level of perioperative complications. The long-term prognosis is good only in patients subjected to the destination therapy. Independent factors that influence the prognosis are a higher mean gradient on the aortic valve prior to the procedure, female sex, presence of neurological disorders, peripheral artery disease, efficacy of the procedure, presence of arterial hypertension, and performance of the target treatment. Unfavorable longterm results of the balloon aortic valvuloplasty speak in favor of the fact that this method should be a bridge to the percutaneous implantation of the aortic valve or its replacement.

\section{Acknowledgments}

The publication has been prepared on behalf of CardValve Consortium, established for the InFlow project and funded by the National Centre for Research and Development. Project number: StrategMed 1/233166/6/ NCBR/2014; project title: Development and introduction of the first Polish low-profile aortic valve implanted percutaneously.

\section{Conflict of interest}

The authors declare no conflict of interest.

\section{References}

1. Davidson C, Harrison J, Leithe M. Failure of balloon aortic valvuloplasty to result in sustained clinical improvement in patients with depressed left ventricular function. Am J Cardiol 1990; 65: 72-7.

2. Desk R, Williams L, Health K. Percutaneous balloon aortic valvuloplasty. Acute and 30-day follow-up results in 674 patients from the NHLBI Balloon Valvuloplasty Registry. Circulation 1991; 84: 2383-97.

3. McKay RG. The Mansfield Scientific Aortic Valvuloplasty Registry: overview of acute hemodynamic results and procedural complications. J Am Coll Cardiol 1991; 17: 485-91.

4. Vahanian A, Alfieri O, Andreotti F, et al. Guidelines on the management of valvular heart disease (version 2012). Eur Heart J 2012; 33: 2451-96.

5. Kappetein AP, Head SJ, Généreux P, et al. Updated standardized endpoint definitions for transcatheter aortic valve implantation: the Valve Academic Research Consortium-2 consensus document. J Thorac Cardiovasc Surg 2013; 145: 6-23.

6. Ben-Dor I, Maluenda G, Dvir D, et al. Balloon aortic valvuloplasty for severe aortic stenosis as a bridge to transcatheter/surgical aortic valve replacement. Catheter Cardiovasc Interv 2013; 82: 632-7.

7. Klein A, Lee K, Gera A. Long-term mortality, cause of death, and temporal trends in complications after percutaneous aortic balIoon valvuloplasty for calcific aortic stenosis. J Interv Cardiol 2006; 19: 269-76.

8. Lieberman EB, Bashore TM, Hermiller JB, et al. Balloon aortic valvuloplasty in adults: failure of procedure to improve long-term survival. J Am Coll Cardiol 1995; 26: 1522-8.

9. Eltchaninoff $H$, Durand E, Borz B, et al. Balloon aortic valvuloplasty in the era of transcatheter aortic valve replacement: acute and long-term outcomes. Am Heart J 2014; 167: 235-40.

10. Ben-Dor I, Pichard AD, Satler LF, et al. Complications and outcome of balloon aortic valvuloplasty in high-risk or inoperable patients. JACC Cardiovasc Interv 2010; 3: 1150-6.

11. Reeder GS, Nishimura RA, Holmes DR. Patient age and results of balloon aortic valvuloplasty: the Mansfield Scientific Registry experience. The Mansfield Scientific Aortic Valvuloplasty Registry Investigators. J Am Coll Cardiol 1991; 17: 909-13.

12. Agarwal A, Kini AS, Attanti $S$, et al. Results of repeat balloon valvuloplasty for treatment of aortic stenosis in patients aged 59 to 104 years. Am J Cardiol 2005; 95: 43-7.

13. Saia F, Marrozzini C, Ciuca C, et al. Emerging indications, in-hospital and long-term outcome of balloon aortic valvuloplasty in the transcatheter aortic valve implantation era. Eurolntervention 2013; 8: 1388-97.

14. Daniec M, Sorysz D, Dziewierz A, et al. In-hospital and long-term outcomes of percutaneous balloon aortic valvuloplasty with concomitant percutaneous coronary intervention in patients with severe aortic stenosis. J Interv Cardiol 2018; 31: 60-7.

15. Leon MB, Piazza N, Nikolsky E, et al. Standardized endpoint definitions for transcatheter aortic valve implantation clinical trials: a consensus report from the Valve Academic Research Consortium. Eur Heart J 2011; 32: 205-17.

16. Daniec M, Nawrotek B, Sorysz D, et al. Acute and long-term outcomes of percutaneous balloon aortic valvuloplasty for the treatment of severe aortic stenosis. Catheter Cardiovasc Interv 2017; 90: 303-10. 
17. Arsalan M, Khan S, Golman J. et al. Balloon aortic valvuloplasty to improve candidacy of patients evaluated for transcatheter aortic valve replacement. J Interv Cardiol 2018; 31: 68-73.

18. Saia F, Marrozzini C, Moretti C, et al. The role of percutaneous balloon aortic valvuloplasty as a bridge for transcatheter aortic valve implantation. Eurolntervention 2011; 7: 723-9.

19. Daniec M, Dziewierz A, Sorysz D, et al. Sex-related differences in outcomes after percutaneous balloon aortic valvuloplasty. J Invasive Cardiol 2017; 29: 188-94.

20. Eugène $M$, Urena $M$, Abtan J, et al. Effectiveness of rescue percutaneous balloon aortic valvuloplasty in patients with severe aortic stenosis and acute heart failure. Am J Cardiol 2018; 121: 746-50. 